\title{
Lésion de la branche médiale du nerf fibulaire profond : données cliniques, échographiques et thérapeutiques de 78 pieds
}

Maurice Bouysset, ${ }^{1,2}$ Delphine Denarié, ${ }^{3}$ Thierry Tavernier, ${ }^{4}$ Jean Depassio, ${ }^{5}$ Jean-Yves Coillard, ${ }^{6}$ Daniel Boublil, ${ }^{6}$ Jacques Guy Tebib, ${ }^{1,2^{*}}$ Fabienne Coury, ${ }^{1,2^{*}}$

${ }^{1}$ Hospices Civils de Lyon, Centre Hospitalier Lyon Sud, Service de Rhumatologie, 69495

Pierre-Bénite, France

${ }^{2}$ Université Lyon 1, 69000 Lyon, France

${ }^{3} \mathrm{CHU}$ Nord, Service de rhumatologie, 42270 Saint Priest en Jarez, France

${ }^{4}$ Clinique de la Sauvegarde, Service de Radiologie, 69000 Lyon, France

${ }^{5} 11$ montée de Verdun, 69160 Tassin la Demi-Lune, France

${ }^{6}$ Clinique du Parc, 69000 Lyon, France

* contribution égale

\section{Auteur correspondant :}

Fabienne Coury

Service de Rhumatologie

Centre Hospitalier Lyon Sud

165 Chemin du Grand Revoyet

69495 Pierre-Bénite, France

Phone: +33 (0)4 78865695

Fax: +33(0)4 72618881

Email : fabienne.coury@free.fr; fabienne.coury-lucas@chu-lyon.fr 


\section{RESUME}

Objectif : Étudier les symptômes de l'atteinte de la branche médiale du nerf fibulaire profond et l'amélioration des signes fonctionnels après traitement.

Méthodes : Des patients avec une histoire clinique de troubles sensitifs d'irritation du nerf fibulaire profond ont été successivement sélectionnés par 3 praticiens orientés en pathologie du pied. Chaque pied présentait au moins un point gâchette sur le trajet du nerf à l'examen physique. Si l'atteinte était confirmée par l'électromyogramme, le patient était inclus dans l'étude. Une recherche de troubles de la sensibilité cutanée de la première commissure intermétatarsienne, une radiographie de profil en charge et une échographie étaient ensuite réalisées pour chaque pied.

Résultats : Parmi les 78 pieds inclus présentant une atteinte de la branche médiale du nerf fibulaire profond confirmée par électroneuromyographie, 59 (76\%) présentaient un trouble sensitif de la première commissure intermétatarsienne et $34(44 \%)$ un pied creux. L'échographie retenait au moins un conflit avec le nerf sur 40 pieds (51\%). Trente des 59 pieds $(51 \%)$ présentant un trouble sensitif de la première commissure intermétatarsienne présentaient également un conflit en échographie. Le traitement conservateur a été efficace sur 68/78 pieds, dont 31/40 (78\%) avec conflit. Dix pieds sur $78(13 \%)$ ont eu une libération chirurgicale du nerf et 8/10 pieds ont été améliorés par la chirurgie.

Conclusion: Ces résultats doivent inciter en pratique le rhumatologue à rechercher à l'examen clinique une souffrance de la branche médiale du nerf fibulaire profond.

Mots clés : pied, branche médiale du nerf fibulaire profond 


\section{Introduction}

En 1968, Marinacci a dénommé « syndrome du tunnel tarsien antérieur » la souffrance du nerf fibulaire profond (NFP) au pied et à la cheville [1]. Depuis, de nombreuses observations ont été publiées sur le sujet et Dellon a étudié en détail 20 cas de lésions de la branche médiale de ce nerf fibulaire profond (MNFP) [2].

Le nerf fibulaire profond est une des deux branches terminales du nerf fibulaire commun. II naît à la tête de la fibula puis chemine entre les muscles tibial antérieur et long extenseur de I'hallux. Le tendon de ce dernier muscle surcroise le NFP à la cheville, puis le NFP chemine sous le rétinaculum inférieur des extenseurs et entre dans l'espace nommé tunnel tarsien antérieur [1]. Dans ce tunnel cheminent l'artère dorsale du pied et sa veine, le NFP, et les tendons des muscles tibial antérieur, long extenseur de l'hallux, long extenseur des orteils et troisième fibulaire. Le NFP se divise en branches latérale et médiale en proximal de la tête du talus, plus rarement la division est un peu plus distale [3 45 6 5 ]. La branche latérale est motrice pour le muscle court extenseur des orteils. La MNFP continue sur le bord médial du dos du pied puis passe sous le faisceau tendineux médial du muscle court extenseur des orteils. Cette MNFP est purement sensitive et innerve la peau de la première commissure intermétatarsienne.

La lésion de la MNFP doit être connue car elle peut simuler les symptômes d'une arthrose tarsienne, d'une compression du nerf fibulaire commun à la tête de la fibula ou d'une radiculopathie L5. Sur des patients avec confirmation diagnostique de lésion de la MNFP par l'électroneuromyogramme (ENMG), nous avons ainsi évalué l'importance de certains signes cliniques, comme la recherche de points gâchettes et de troubles sensitifs, et des signes échographiques. La radiographie de profil en charge des pieds étudiés a permis de déterminer les éventuels troubles statiques. L'amélioration des signes fonctionnels après traitement a également été évaluée.

\section{Méthodes}

\subsection{Patients}

Des pieds présentant des douleurs et des symptômes évocateurs de lésion de la MNFP tels que des sensations de brûlure, des paresthésies, des sensations électriques sur la face dorso-médiale du pied, ont été successivement examinés dans les consultations de 3 praticiens dont la pratique est orientée vers la prise en charge de la pathologie du pied et de la cheville (MB, JYC, DB). Les traitements par voie orale, antalgiques et anti-inflammatoires 
surtout, parfois toniques veineux, et/ou des orthèses plantaires n'avaient pas apporté d'amélioration. L'anamnèse a recherché un facteur déclenchant local (traumatique ou microtraumatique). La détection de l'atteinte de la MNFP s'est basée sur les recommandations de Mann et Baxter pour l'étude des lésions du nerf tibial [7]. Une première recherche des points gâchettes, reproduisant les symptômes spontanément ressentis, a été effectuée par chaque praticien recruteur pour permettre l'inclusion [7]. Si un point gâchette était présent un ENMG était réalisé pour confirmer le diagnostic de lésion de la MNFP et si confirmation le patient était inclus dans l'étude [7]. Le recrutement des patients a débuté en décembre 2007 et la dernière évaluation des résultats thérapeutiques a été effectuée en novembre 2012. L'approbation du comité d'éthique n'était pas requise conformément à la politique de notre institution.

Les patients inclus dans l'étude ont été soumis à un protocole d'évaluation clinique précise pratiquée par un praticien (MB) [2, 8, 9]. Une radiographie de profil en charge du pied et une échographie (TT) ont été réalisées. Selon les recommandations de Haddad, la recherche de «points gâchettes » a été effectuée par palpation localisée appuyée durant 30 secondes, la pression utilisée correspondant à l'apparition de la décoloration sous l'ongle du pouce qui appuie [8]. L'irradiation proximale ou distale de la douleur sur le trajet du nerf reproduisait les symptômes spontanément ressentis [8]. Chaque pied examiné présentait au moins un point gâchette [2, 3, 10]. Les points gâchettes observés incluaient le col distal du talus qui correspond au tunnel tarsien antérieur lui-même, l'os naviculaire et la surface à la jonction des bases des $1^{\text {er }}$ et $2^{\mathrm{e}}$ métatarsiens avec les cunéiformes (Figure 1) [2, 3, 5, 10]. Aucun symptôme pouvant évoquer une autre neuropathie ne devait être observé au pied ou à la cheville. II ne devait pas y avoir de contexte de polyneuropathie générale, ni de patient ayant un contexte de droit à réparation juridique. Étaient considérés ici les troubles sensitifs par lésion du NFP à l'intérieur du tunnel tarsien antérieur lui-même et sur sa branche médiale. Une recherche attentive d'hypoesthésie ou d'anesthésie de la première commissure intermétatarsienne était effectuée [2, 3, 7, 9, 11]. Son importance est soulignée par Dellon. [2]. Ces troubles sensitifs étaient retenus si une diminution de la perception au toucher léger $[2,3,5]$ et à l'effleurement de la première commissure intermétatarsienne avec un monofilament [6] était observée. Un monofilament $10 \mathrm{G}$ était utilisé avec d'abord 3 effleurements sur les aires explorées, puis après un délai d'environ une minute, une $2^{\mathrm{e}}$ série de 3 effleurements sur les mêmes zones, et à nouveau après une minute une $3^{\mathrm{e}}$ série de 3 effleurements avec le monofilament. L'appréciation du patient après ce $3^{\mathrm{e}}$ passage établissait le diagnostic clinique de lésion de la MNFP si un trouble sensitif était observé. Le toucher léger de la première commissure interorteils était effectué dans les mêmes conditions avec la pulpe du majeur. Ces troubles sensitifs étaient recherchés en comparaison avec la zone supéro-latérale du même pied, qui est innervée par le nerf 
fibulaire superficiel, et avec le pied controlatéral quand cela était possible en cas d'atteinte unilatérale $[2,11]$.

L'électromyogramme a été effectué par un praticien expérimenté (JD). Toute anomalie sensitive observée sur le NFP servait de référence pour sélectionner les cas observés.

Les études de conduction motrice (MCVs) étaient effectuées avec une bande passante $3 \mathrm{~Hz}$ -

$3 \mathrm{kHz}$. Les stimulations étaient successivement réalisées sur la face antérieure de la ligne bimalléolaire, sous et au-dessus la tête de la fibula, avec l'électrode de réception de surface placée sur le muscle court extenseur des orteils.

Les études de conduction sensitive (SCVs) étaient effectuées en orthodromique avec une bande passante $20 \mathrm{~Hz}-3 \mathrm{kHz}$. L'électrode de réception de surface était placée sur le bord antérieur et médial de la cheville et une stimulation bipolaire était pratiquée entre le $1^{\mathrm{er}}$ et le $2^{e}$ métatarsiens. La distance entre électrodes de stimulation et de réception variait entre 8 et $12 \mathrm{~cm}$.

La radiographie de profil en charge des pieds recherchait la présence de pieds creux et de pieds plats. Elle permettait de calculer l'angle de l'arche médiale formé par le croisement de 2 lignes. La première joint le point le plus bas du sésamoïde médial et le point le plus bas de l'articulation talo-naviculaire. La seconde ligne joint ce point précédent avec le point le plus bas du calcanéus [12]. Les troubles statiques, pied plat $\left(>130^{\circ}\right)$ et pied creux $\left(<118^{\circ}\right)$, étaient notifiés. L'évaluation clinique et la lecture des radiographies ont été effectuées par le même praticien (MB).

L'échographie avec la mobilisation du pied pendant l'examen permettait d'apprécier les conflits sur le nerf. Les échographies ont été réalisées avec des sondes linéaires de haute fréquence (12 MHz et $18 \mathrm{MHz}$ ) ainsi qu'avec une petite sonde « club de golf » de $15 \mathrm{MHz}$. L'étude des nerfs était pratiquée grâce à la technique de l'ascenseur (échographie dynamique) avec déplacement rapide de la sonde de haut en bas et de bas en haut. Le mode B était complété par une analyse en doppler couleur.

L'échographie permettait l'évaluation des synovites, des ténosynovites et de l'inflammation des tissus sous-cutanés. De plus le doppler puissance aidait à faire la distinction entre les structures vasculaires et les nerfs. Les échographies ont été réalisées par le même praticien (TT).

\subsection{Traitement}

Chaque patient a bénéficié pendant 6 mois d'un traitement conservateur qui visait surtout à éviter compression et tension du nerf en cause : diminution du niveau d'activité physique, chaussage non compressif pour diminuer la pression de la chaussure sur la face dorsale du pied, pas de talons hauts [2, 3, 4], correction d'une hyperpronation par orthèses plantaires 
[3], utilisation de protecteurs amortissants comme une couche de coton ou le port de chaussures avec languette amortissante pouvant aider à protéger la zone fragilisée autour du nerf, thérapeutiques anti-œdémateuses parfois utiles en cas d'œdème local [9], port d'attelles de repos nocturnes évitant la flexion plantaire importante de la cheville qui entraîne compression et étirement du nerf sur l'os naviculaire $[3,5,10]$. Les injections de corticoïdes étaient utilisées en dernier recours sur les points gâchettes observés [5, 9]. Si après 6 mois malgré le traitement conservateur, la gêne fonctionnelle à l'interrogatoire restait importante, l'indication chirurgicale était proposée en raison de résultats souvent bénéfiques dans les cas publiés $[3,4,15]$. Le patient était orienté vers un des chirurgiens orthopédistes impliqués dans l'étude (JYC et DB). Les résultats de la libération chirurgicale étaient observés entre 6 à 12 mois suivant chaque intervention par un interrogatoire du patient qui évaluait la gêne fonctionnelle due à la douleur. Ces résultats étaient classés en 5 stades: 0, pas d'amélioration ; grade 1, de 0 à $30 \%$ d'amélioration soit un effet médiocre ; grade 2, de 31 à $59 \%$ d'amélioration soit résultat moyen ; grade 3, de 60 à $79 \%$ d'amélioration, soit bon résultat ; grade 4, de 80 à $100 \%$ d'amélioration, soit très bon résultat du traitement.

\section{Résultats}

Soixante-dix-huit pieds issus de 72 patients ayant une anomalie sensitive de la MNFP à l'électromyogramme ont été inclus. Les caractéristiques des patients sont détaillées dans le tableau 1. Parmi ces 78 pieds, 59 (76\%) avaient une hypoesthésie ou une anesthésie du $1^{\mathrm{er}}$ espace inter-métatarsien. Les radiographies montraient des troubles statiques pour 47 pieds $(60 \%)$ avec $34 / 78$ pieds creux (44\%) et 13/78 (16\%) pieds plats. L'échographie a permis de déceler un conflit avec le nerf sur $40 / 78$ pieds (51\%). Ces conflits étaient dus à des ostéophytoses (32 cas) (Figure 2), à un kyste synovial issu de l'articulation talo-naviculaire (3 cas), à un kyste talo-naviculaire et à une ostéophytose à la fois (3 cas), ou encore à une ostéophytose associée à une ténosynovite du long extenseur des orteils (1 cas) (tableau 2). Parmi les 59 pieds avec hypoesthésie, 30 (51\%) présentaient un conflit. Le traitement conservateur a été efficace pour $68 / 78$ pieds (87\%) au total, et $31 / 40$ pieds $(78 \%)$ présentant un conflit à l'échographie ont été améliorés par le traitement conservateur. Seulement $10 / 78$ pieds (13\%) ont nécessité une neurolyse de la MNFP. Neuf de ces pieds opérés présentaient un trouble sensitif et 9 avaient un conflit décelé par l'imagerie. Le pied sans trouble sensitif à l'examen physique provenait d'un patient présentant une atteinte bilatérale de la MNFP sur l'électromyogramme. L'indication chirurgicale a été retenue pour les 2 pieds chez ce patient du fait d'un conflit par ostéophytose. Dans 8 cas, la chirurgie a donné de bons ou très bons résultats (2 d'entre eux ont cependant subi une seconde intervention chirurgicale aux $3^{e}$ et $6^{e}$ mois pour fibrose). Une patiente avec des résultats 
médiocres sur la douleur présentait également une fibromyalgie. Une autre avec des suites défavorables avait subi un traumatisme majeur sur le dos du pied avec possible lésion anatomique du nerf.

\section{Discussion}

Toute compression du nerf peut engendrer des symptômes de lésion de la MNFP [2, 4, 5]. Dans la lésion de la MNFP, certains symptômes sont dus à des facteurs extrinsèques : à la cheville et sur la face dorsale du pied la MNFP est particulièrement sujette aux traumatismes du fait de sa proximité avec le plan osseux et en raison d'une faible épaisseur tissulaire protectrice [4]. Dans ces conditions, les compressions du nerf surviennent à la suite de traumatismes directs ou indirects, qu'il s'agisse de fractures, de subluxations, d'entorses de cheville, ou de contusions. [1, 4, 13]. Il en est de même pour les microtraumatismes sur le dos du pied, qui sont dus à des chaussures trop serrées par exemple. L'œdème sous-cutané au pied et à la cheville peut provoquer une compression dans la chaussure [13]. L'imagerie peut observer certaines causes de compression : l'ostéophytose tibiale antérieure [14], plus souvent l'ostéophytose dorsale du tarse $[4,10,15]$ et des kystes synoviaux issus d'articulations du tarse comme il en a été observé dans notre étude [14]. Nous avons observé une ténosynovite du tendon du muscle long extenseur des orteils sur un pied creux important chez un marathonien. D'autres étiologies sont classiques : os intermetatarseum [16-17], anévrismes [14], ganglions dans le tunnel tarsien antérieur [4], thrombose de l'artère dorsale du pied $[6,18]$. La MNFP passe sous le faisceau médial du tendon du muscle court extenseur des orteils qui peut la comprimer [9, 19]. L'os intermetatarseum est un os accessoire du pied relativement rare, dont la fréquence est estimée entre 1,2 et $14 \%$ dans la littérature. Habituellement situé entre les bases des $1^{\mathrm{er}}$ et $2^{\mathrm{e}}$ métatarsiens et asymptomatique dans la plupart des cas [17], il n'a pas été observé chez nos patients. La présence de l'os intermetatarseum prédispose à une lésion de la MNFP qui peut apparaître lors de traumatismes ou de microtraumatismes. Cette prédisposition est vérifiée pour l'os intermetatarseum mais aussi dans le cas du pied creux [2, 3, 5] (cf infra). Elle peut également être considérée pour l'ostéophytose dorsale du tarse qui précède l'apparition des symptômes d'irritation de la MNFP.

Dans $51 \%$ des cas de lésion de la MNFP, l'échographie a noté la présence d'un conflit. Cette observation souligne pour les $49 \%$ des cas restants la possibilité de compressions du nerf extrinsèques au pied mais aussi d'autres causes que la compression. En l'occurrence, à côté des lésions par compression, il a été observé que l'étirement répété du NFP sur la face dorsale du tarse peut favoriser l'apparition d'un syndrome du tunnel tarsien antérieur [20]. Les talons hauts provoquent à la fois un étirement et une compression du nerf contre l'os 
naviculaire par les mouvements de flexion plantaire et d'hyperextension des orteils qu'ils provoquent $[1,3,6,13,21]$. Ces mouvements expliquaient pour Liu les symptômes d'un danseur de ballet [4]. Les contraintes mécaniques dues aux troubles de la statique du pied sont un facteur prédisposant [2, 3, 5]. Le pied creux est plus à risque car l'arche longitudinale avec proéminence des articulations talo-naviculaire et cunéo-naviculaire étire le rétinaculum inférieur et provoque une compression. L'affaissement de l'arche médiale peut également être en cause [3]. Tout élément qui limite la mobilité du nerf peut engendrer une lésion [4], notamment une fibrose cicatricielle avec disparition du tissu aréolaire protecteur autour du nerf [3].

Théoriquement, la présence d'un conflit avec le nerf aurait laissé présager un résultat péjoratif du traitement conservateur. Cependant dans $78 \%$ des cas de conflit à l'échographie une amélioration a été obtenue par le traitement conservateur. Les signes fonctionnels ont disparu alors que la cause du conflit n'a pas disparu. Ce résultat souligne l'intérêt d'une attitude thérapeutique d'abord conservatrice qui s'avère souvent efficace dans notre échantillon.

Le diagnostic différentiel de neuropathie du NFP doit exclure d'autres douleurs de la face dorsale du pied, qu'elles soient d'origine arthrosique, ou dues à des tendinopathies et exclure une lésion du nerf fibulaire superficiel qui peut d'ailleurs être associée (déficit de sensibilité à la face dorsale du pied exceptée la première commissure interorteils), une lésion du nerf fibulaire commun (perte de force des muscles tibial antérieur et long extenseur des orteils). Une radiculopathie L5 et une polyneuropathie doivent également être éliminées. Ces derniers éléments du diagnostic différentiel soulignent l'importance de l'ENMG qui permet d'éliminer d'autres neuropathies.

Un des pieds opérés ne présentait pas de troubles sensitifs. Le patient concerné présentait une atteinte bilatérale de la MNFP à l'ENMG. Cette observation suggérait que l'hypoesthésie apparaissait manifeste sur la plus atteinte des 2 MNFP concernées, et que le pied controlatéral avec une MNFP moins lésée présentait des troubles sensitifs moindres à l'examen physique.

\section{Remerciements}

Les auteurs remercient le Dr Nadine CHARLES pour ses conseils.

\section{Déclaration de liens d'intérêts}

Les auteurs déclarent ne pas avoir de liens d'intérêt. 


\section{References}

1. Marinacci AA. Neurological syndromes of the tarsal tunnels. Bull Los Angeles Neurol Soc 1968;33:90-100.

2. Dellon AL. Deep peroneal nerve entrapment on the dorsum of the foot. Foot Ankle 1990;11:73-80.

3. Borges LF, Hallett M, Selkoe DJ, Welch K. The anterior tarsal tunnel syndrome. Report of two cases. J Neurosurg 1981;54:89-92.

4. Liu Z, Zhou J, Zhao L. Anterior tarsal tunnel syndrome. J Bone Joint Surg Br 1991;73:4703.

5. Flanigan RM, DiGiovanni BF. Peripheral nerve entrapments of the lower leg, ankle, and foot. Foot Ankle Clin 2011;16:255-74.

6. Andresen BL, Wertsch J, Stewart WA. Anterior tarsal tunnel syndrome. Arch Phys Med Rehabil 1992;73:1112-7.

7. Mann RA, Baxter DE. Diseases of the nerve. In: Mann RA, Coughlin JC, ed. Surgery of the foot and ankle. St Louis (MO): Mosby;1993, 554-8.

8. Haddad SL. Compressive neuropathies of the foot and ankle. In: Myerson MS, ed. vol 2, 808-33.

9. Kanbe K, Kubota H, Shirakura K, Hasegawa A, Udagawa E. Entrapment neuropathy of the deep peroneal nerve associated with the extensor hallucis brevis. J Foot Ankle Surg 1995; 34:560-2.

10. Edlich HS, Fariss BL, Phillips VA, et al. Talotibial exostoses with entrapment of the deep peroneal nerve. J Emerg Med 1987;5:109-13.

11. Milants C, Wang FC, Gomulinski L, et al. The anterior tarsal tunnel syndrome: a case report. Revue médicale de Liège 2015;70:400-4.

12. Bouysset M, Tebib JG, Weil E, Lejeune E, Bouvier M. Deformation of the adult rheumatoid rearfoot. A radiographic study. Clin Rheumatol 1987;6:539-44.

13. Krause KH, Witt T, Ross A. The anterior tarsal tunnel syndrome. J Neurol 1977;217:6774.

14. Kim JY, Ihn YK, Kim JS, Chun KA, Sung MS, Cho KH. Non-traumatic peroneal nerve palsy: MRI findings. Clin Radiol 2007;62:58-64.

15. Huang KC, Chen YJ, Hsu RW. Anterior tarsal tunnel syndrome: case report. Changgeng Yi Xue Za Zhi 1999;22:503-7.

16. Knackfuss IG, Giordano V, Nogueira M, Giordano M. Compression of the medial branch of deep peroneal nerve, relieved by excision of an os intermetatarseum. A case report. Acta Orthop Belg 2003;69:568-70.

17. Nakasa T, Fukuhara K, Adachi N, Ochi M. Painful os intermetatarseum in athletes: report of four cases and review of the literature. Arch Orthop Trauma Surg 2007; 127:261-4. 
18. Gani N, Khan HA, Kamal Y, Farooq M, Jeelani H, Shah AB, Anterior tarsal syndrome with thrombosed dorsalis pedis artery: A case report. ArchTrauma Res 2015;4:e21738.

19. Reed SC, Wright CS. Compression of the deep branch of the peroneal nerve by the extensor hallucis brevis muscle: a variation of the anterior tarsal tunnel syndrome. Can $\mathrm{J}$ Surg 1995;38:545-6.

20. Akyuz G, Us O, Turan B, Kayhan O, Canbulat N, Yilmar IT. Anterior tarsal tunnel syndrome. Electromyogr Clin Neurophysiol 2000;40:123-8.

21. Posas HN, Rivner MH. Deep peroneal sensory neuropathy. Muscle Nerve 1992;15:7456. 


\section{LEGENDES}

Figure 1: Points gâchettes sur la branche médiale du nerf fibulaire profond. TTA, tunnel tarsien antérieur. $\mathrm{N}$, os naviculaire. BM1M2, bases des $1^{\mathrm{er}}$ et $2^{\mathrm{e}}$ métatarsiens. 1 , nerf fibulaire profond. 2, branche médiale du nerf fibulaire profond. 3, branche latérale du nerf fibulaire profond. 4, branches issues de la branche latérale dont l'une pénètre dans le sinus du tarse. 5 , branche récurrente pour la capsule antéro-latérale de la cheville. 6 , nerf fibulaire superficiel (d'après "Pathologie ostéo-articulaire du pied et de la cheville " de la clinique aux examens complémentaires, Sauramps Médical Ed. Bouysset M, Delmi M, Morvan G 2014).

Figure 2: Radiographie du pied de profil en charge: arthrose tarso-métatarsienne avec importante ostéophytose. 


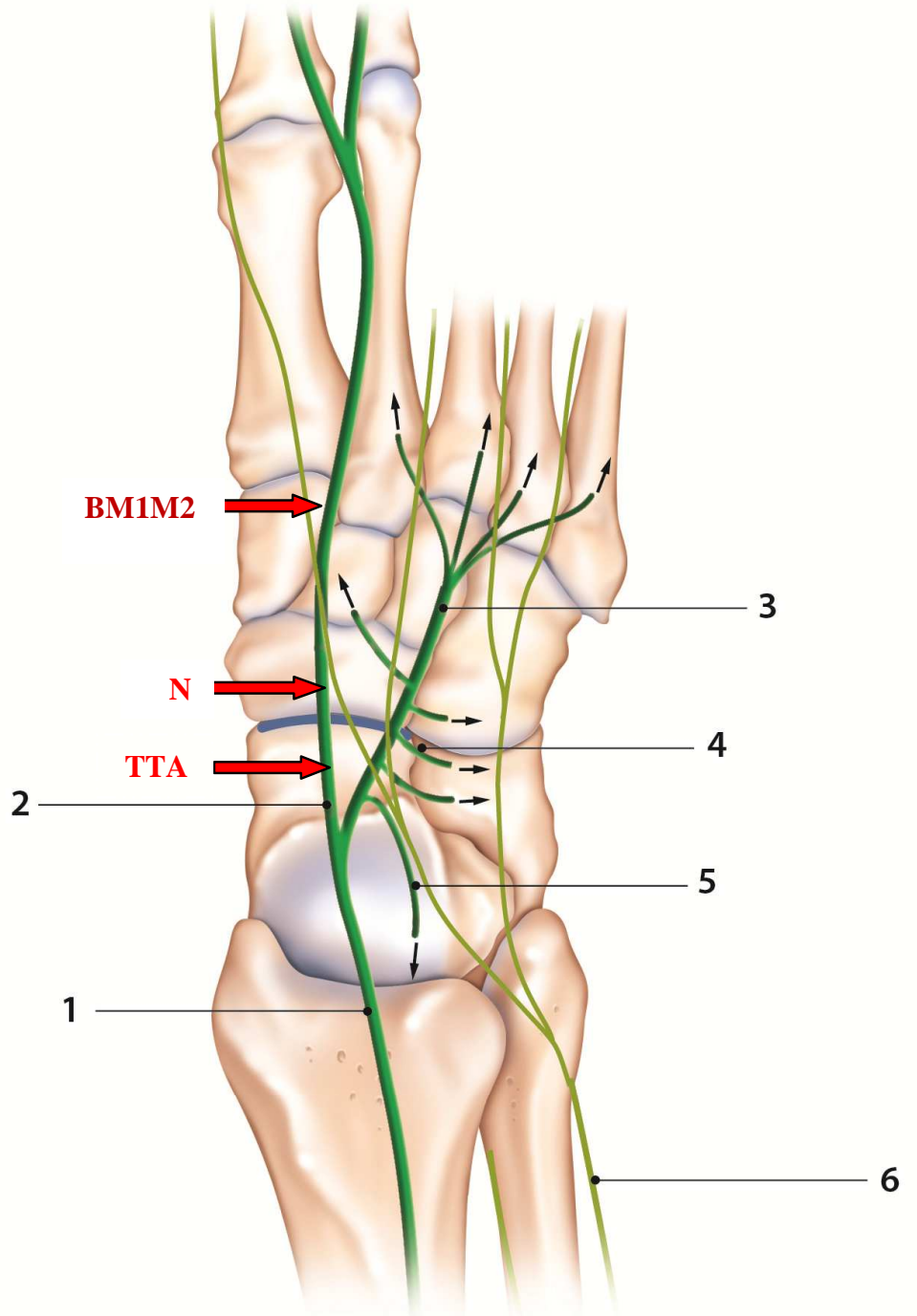




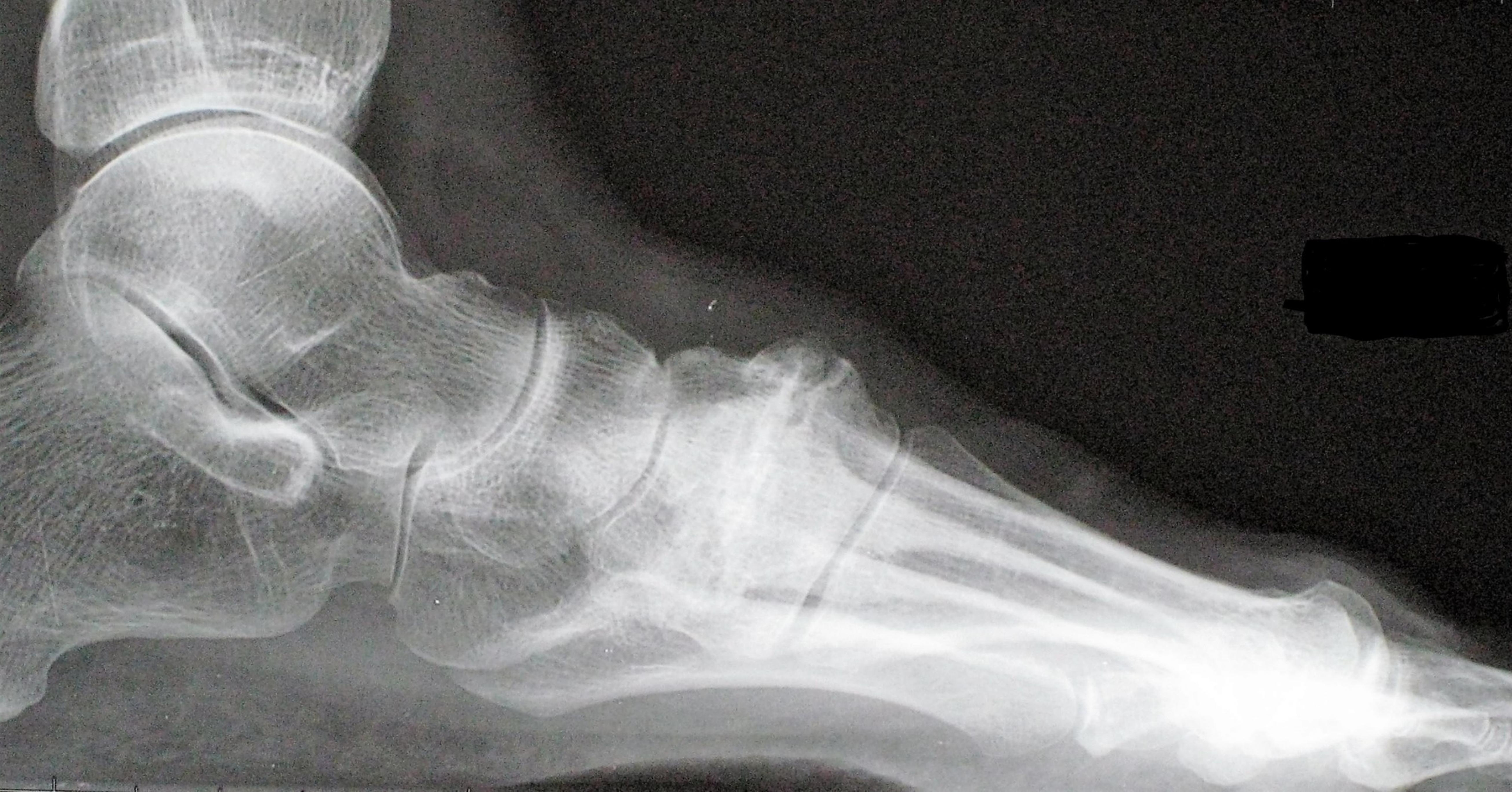




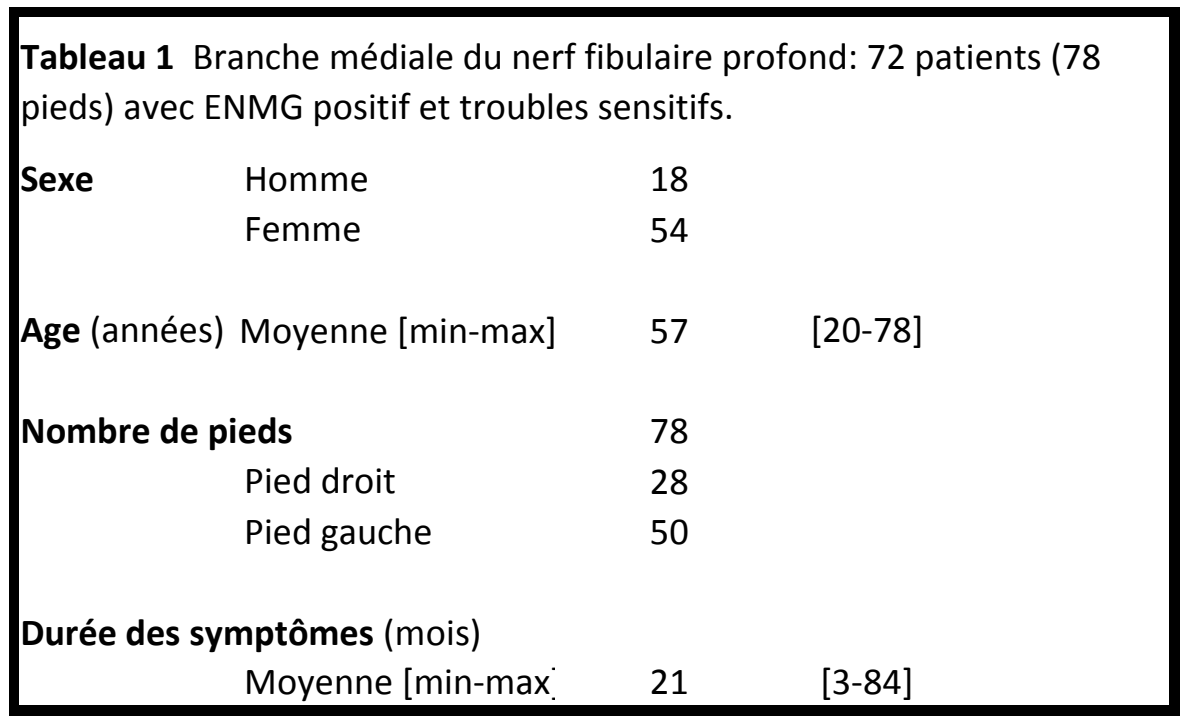




\section{Tableau 2}

78 pieds avec lésion de la branche médiale du nerf fibulaire profond au pied et à la cheville avec

ENMG positif.

Troubles sensitifs du $1^{\mathrm{er}}$ espace inter-métatarsien

Troubles statiques

Pieds creux 34

Pieds plats 13

Conflits observés sur le nerf (échographie) $\quad 40$

Conflits osseux isolés 32

Conflits avec kyste synovial $\quad 4$

Conflits osseux et avec kyste synovial 3

Conflits osseux et avec LEO (1) $\quad 1$

(1) LEO : ténosynovite du tendon long extenseur des orteils. 